\title{
El ABPrj como estrategia pedagógica aplicada en un curso de programación orientada a objetos
}

\section{The ABPrj as a pedagogical strategy applied in an object oriented programming course}

\begin{abstract}
Luis Adrian Lasso-Cardona
Universidad del Valle - sedes Buga y Tuluá, Colombia

Objetivo: Evaluar el grado de percepción que tuvieron los alumnos con respecto al uso del ABPrj como herramienta pedagógica usada en el curso de Programación Orientada a Objetos, dictada en las sedes de Buga y Tuluá de la Universidad del Valle. Método: La investigación se realizó bajo un enfoque descriptivo-cuantitativo. El enfoque descriptivo conceptualizo el tema permitiendo evidenciar la importancia del ABPrj desde el punto de vista pedagógico y de formación de competencias en futuros egresados. El enfoque cuantitativo permitió medir los datos recolectados de la encuesta aplicada, la cual fue diseñada con base en el Cuestionario de Efectividad del Uso de Metodologías de Participación Activa. El estudio se realizó en cinco fases: 1) Recolección de información, 2) Construcción del marco teórico, 3) Diseño y aplicación de la encuesta, 4) Análisis de datos recolectados, y 5) Conclusiones. Resultados: Se obtuvo una calificación promedio de 4.5 pts., equivalente al $90 \%$ de aprobación. Se evidencio una correlación entre la edad y el promedio de la evaluación, alcanzando su mayor diferencia en los estudiantes de Tecnología con un $16.61 \%$. Discusión y Conclusiones: Se confirmó la hipótesis del investigador, en cuanto a que no existieron diferencias muy marcadas en los estudiantes de Tecnología e Ingeniería respecto a la evaluación de la estrategia implementada.
\end{abstract}

\section{Resumen}

Palabras clave: Aprendizaje activo, método de aprendizaje, enseñanza y formación, competencia profesional, programación informática

\begin{abstract}
Objective: To evaluate the degree of perception that students had regarding the use of the ABPrj as a pedagogical tool used in the Object Oriented Programming course, taught at the Buga and Tuluá campuses of the Universidad del Valle. Method: designed based on the Questionnaire on the Effectiveness of the Use of Active Participation Methodologies. The study was carried out in five phases: 1) Collection of information, 2) Construction of the theoretical framework, 3) Design and application of the survey, 4) Analysis of collected data, and 5) Conclusions. Results: An average score of 4.5 pts was obtained, equivalent to $90 \%$ approval. A correlation between age and the average of the evaluation was evidenced, reaching its greatest difference in the Technology students with $16.61 \%$. Discussion and Conclusions: The researcher's hypothesis was confirmed, in that there were no very marked differences in the students of Technology and Engineering regarding the evaluation of the implemented strategy.
\end{abstract}

Keywords: Activity learning, learning methods, teaching and training, occupational qualifications, computer programming.
Open Access:

ISSN: 0124-2121

E-ISSN: $2665-2420$

Editor:

Dhayana Fernández Matos

TIPOLOGÍA DE ARTÍCULO
Copyright @

By Educación y Humanismo

Correspondencia:

luis.lasso@correounivalle.edu.co

Recibido: $30-07-2021$

Aceptado: 29-11-2021

En línea desde: $14-01-2022$

Cómo citar este artículo (APA): Lasso-Cardona, L. (2022). El ABPrj como estrategia pedagógica aplicada en un curso de programación orientada a objetos. Educación y Humanismo, 24(42), 21-45. https://doi.org/10.17081/eduhum.24.42.4822 


\section{Introducción}

En la actualidad la programación de computadoras es una de las áreas a nivel mundial de mayor demanda en el ámbito laboral, debido al auge en el desarrollo de nuevas tecnologías que requieren de programas informáticos para su utilización y gestión. Es por ello que cada vez más se requiere de personal calificado en diversos campos de la computación y la matemática, que ayuden a suplir las necesidades de una sociedad cada vez más moderna y digital (García et al., 2020).

Es este escenario, uno de los mayores retos a los cuales se enfrentan las facultades de ingeniería está relacionada con la utilización de metodologías de enseñanza que verdaderamente logren en los estudiantes aprendizajes significativos y competencias, y que desde una postura holística, le den al ingeniero, independiente de su perfil, conocimiento suficiente acerca de las ciencias básicas, una formación integral comprometida con la sociedad y el medio ambiente, además de desarrollar un pensamiento lógico, científico y sistémico, que le permitan construir soluciones enmarcadas en un contexto ético (Capote, Rizo y Bravo, 2016). Particularmente, en la implementación de tareas de enseñanza y aprendizaje del área de matemáticas, varios estudios han determinado que las competencias que necesita desarrollar el alumno están relacionadas con el conocimiento adquirido a lo largo de su proceso educativo, lo que implica que debe estar en la capacidad de identificar, comprender y usar ciertos elementos y estructuras cognitivas que van creciendo gradualmente, y que a futuro se convierten en herramientas valiosas para desenvolverse profesionalmente (Gürbüz y Erdem, 2016; Bravo, Arenas y Pineda, 2019; Valbuena, Muñiz, y Berrío, 2020; Olarte, 2020).

Desde inicios del año 2000, la educación universitaria ha sufrido grandes cambios, resultado de la incorporación de nuevos recursos tecnológicos, que han obligado a los docentes a cambiar la metodología usada para impartir sus clases, que habitualmente se fundamenta en un enfoque magistral basado en literatura relacionada con el tema, que sienta las bases teóricas y sobre la cual se aplica una evaluación (Manresa, Gil y Berbegal, 2020). Para Santos (2015), esta metodología solo logra que el docente se enfoque en la evaluación, y los alumnos en prepararse para ser calificados, existiendo una clara definición de papeles entre los alumnos y el docente. Donde el primero es un actor pasivo que muy pocas veces aplica lo aprendido, y el segundo es el responsable de las labores de enseñanza, estableciendo objetivos y generando el contenido educativo (Mora, Carranza y Palíz, 2019).

No obstante, la educación superior está buscando adaptarse a las condiciones actuales de la sociedad, incorporando dentro de los currículos metodologías constructivistas que sean más activas y enfocadas en el estudiante, que logra mayor responsabilidad y tiene la oportunidad de utilizar los saberes obtenidos bajo la metodología de solución de problemas, y donde el maestro se transforma en un orientador y guía del proceso de formación (Fernández-Cabezas, 2017; Durán y Rosado, 2020). 
En concordancia con lo anterior, el Proceso de Bolonia en 1999, estableció las bases para el plan del Espacio Europeo de Educación Superior (EEES), y determino varias pautas entre las cuales se destaca la adopción de varias Instituciones de Educación Superior (IES) de Europa de la metodología de aprendizaje por competencias, el aprendizaje activo y la educación centrada en el estudiante (García y Pérez, 2018). A nivel de la región en décadas recientes, igualmente se ha visto un aumento en el interés por parte de los gobiernos e instituciones en mejorar y actualizar los pensum de todos los niveles educativos, que impulsen la formación habilidades profesionales y blandas demandadas por la comunidad y la economía, y que estas sirvan para solucionar problemas de forma eficiente y sostenible (Rodríguez, Kolmos y Guerra, 2017). En Colombia, el documento CONPES 3809, estipula que se necesita la incorporación de propuestas pedagógicas novedosas y actuales, que acerquen el sistema educativo del país a los estándares internacionales, y que sitúen como actor principal del proceso de formativo al estudiante (CONPES 3809, 2014). Dentro de tales propuestas surgen modelos pedagógicos como el aprendizaje colaborativo, la educación por competencias, el aula invertida, los juegos serios (Serious game) y la Gamificación, que apoyados en las Tecnologías de la Información y la Comunicación (TIC), están orientados a planear nuevas maneras de adquirir conocimiento (León, Martínez y Santos, 2018). Igualmente, metodologías como el Aprendizaje Basado en Problemas (ABP) y el Aprendizaje Basado en Proyectos (ABPrj) son buenas alternativas didácticas a disposición de las IES, ya que ayudan a desarrollar en el estudiante las capacidades exigidas por el Ministerio de Educación (MEN) en cuanto a brindar una formación enfocada en el ser, el saber y el saber hacer, al plantear proyectos transversales e integradores que relacionan los conocimientos adquiridos de diversas áreas del currículo y que a su vez involucran a la sociedad (Barrera, 2017; MEN, s.f).

Si bien es cierto el ABP y ABPrj tienen características similares y están enfocados en resolver un problema, estos se diferencian en que el ABP usa una metodología apoyada en el planteamiento de preguntas acerca del problema, pero no necesariamente involucra el desarrollo de un producto final, solo basta el esquema de un modelo que resuelva el problema. Por su parte el segundo, está dirigido al planteamiento y diseño del problema, que posteriormente se ve reflejado en la implementación de un producto final con ayuda del conocimiento adquirido y posterior (Correa, 2019). En este orden de conceptos, el ABPrj surge como un método de aprendizaje activo constructivista y enfocado en el alumno que afecta de manera positiva la motivación del mismo. Además, está diseñado como una herramienta que ayuda a producir contenidos curriculares de forma interdisciplinaria, por lo tanto, es más una forma de articular un proyecto que involucre los conocimientos logrados en varias asignaturas, que un método para potenciar el modo de impartir una clase magistral (Botella y Ramos, 2019).

Como se mencionó anteriormente, el ABPrj tiene como propósito fundamental poner como centro del proceso pedagógico al estudiante, que desarrolla un proyecto a lo largo del semestre o incluso más, en el cual debe aplicar lo que está aprendiendo y lo aprendido, con la finalidad de que adquiera una formación significativa y prepararlo para afrontar y resolver 
problemas del mundo real, siendo una motivación mayor ya que percibe que lo desarrollado impacta de forma directa y positiva su vida (Martí, Heydrich, Rojas y Hernández, 2010). En este escenario, el objetivo fundamental de la educación superior, particularmente en programas de ingeniería, no solo debe estar enfocado en la aplicación de teorías y métodos científicos, igualmente es necesario involucrar acciones relacionadas con la experiencia, que fomenten capacidades de comunicación y trabajo en equipos multidisciplinares (Sánchez, Ferrero, Conde y Alfonso, 2016).

El ABPrj en la Educación Superior se ha implementado de forma exitosa como herramienta pedagógica por parte de los docentes universitarios de múltiples áreas del conocimiento en distintos niveles educativos, apreciándose en los alumnos mayor motivación en el uso y adquisición de nuevos conocimientos, que mejoran de forma significativa su aprendizaje y el desarrollo de competencias laborales (Maldonado, 2008).

Es por ello que el ABPrj está estrechamente relacionado con el Proyecto Educativo Institucional (PEI) y el Proyecto Educativo del Programa (PEP) de las IES, que se ven en la obligación de ofrecer una enseñanza de calidad acorde con las competencias laborales que exige el entorno (Romero, Forero y Rodríguez, 2018). En este sentido, son varias las IES de Colombia que han involucrado la metodología ABPrj dentro de los PEP de las facultades de Ingeniería. Por ejemplo, la Universidad Nacional, por medio de la Estrategia de Innovación en Educación en Ingeniería está usando el ABP y el ABPrj en los cursos de Seminario de Proyectos I, II y III de la Facultad de Minas (Vélez y Benjumea, 2017, pp. 30-33). Igualmente, la UNAD está implementado el ABPrj en los trabajos de grado de la carrera de Ingeniería de Sistemas con el propósito de incentivar en los estudiantes la investigación para resolver problemas de su entorno (Medina, Quintana y García, 2017).

El PEP de Ingeniería de Sistemas de la Universidad del Valle, establece que los contenidos programáticos de las asignaturas se deben adaptar a métodos de trabajo novedosos, que den solución a problemas reales haciendo uso de metodologías enfocadas a problemas y proyectos (EISC, 2009, pp. 7-9). La Universidad de Pamplona mediante el PEP de Ingeniería de Sistemas plantea la realización de proyectos que se desarrollan a lo largo del semestre y que tiene un entregable final. Tal proyecto debe cubrir los contenidos del pensum, además de incentivar el trabajo en grupo, las actividades propias de la gestión de proyectos, la investigación, la creatividad y el análisis de resolución de problemas, que finalmente ayuden al crecimiento de competencias en los alumnos (Unipamplona, 2018, p. 25).

Igual enfoque propone el PEP de Ingeniería de Sistemas de la UCEVA que traza las directrices para implementar un modelo pedagógico interestructurante de aprendizaje basado en proyectos integradores, donde el estudiante aplique lo aprendido en las asignaturas del pensum (UCEVA, 2013, pp. 17-19). Asimismo, varias investigaciones sobre la aplicación del ABPrj en planes académicos de ingeniería, dan cuenta de la percepción positiva que tienen los estudiantes con respecto a esta estrategia pedagógica (Rodríguez- 
Sandoval, y Cortés-Rodriguez, 2010; Fernández y Duarte, 2013; Angarita, Fernández y Duarte, 2016; López, 2017; Cabedo et al. 2017; Laguado, Ramírez y Hernández, 2018; García, Montaño y Pérez, 2019).

La presente investigación tiene por objetivo evaluar por medio de un cuestionario diseñado en base al propuesto por Carrasco et al., (2015) el grado de percepción que tuvieron los alumnos con respecto al uso de la metodología ABPrj como herramienta pedagógica en el curso de Introducción a la Programación Orientada a Objetos (IPOO) dictada en segundo semestre en las sedes de Buga y Tuluá de la Universidad del Valle, la cual fue cursada por 73 estudiantes en total pertenecientes a los programas de Tecnología en Sistemas de Información (TSI) (34) e Ingeniería de Sistemas (IS) (39), y dictada por el mismo docente. Para los dos cursos se planteó un único proyecto realizado a lo largo del semestre en equipos de trabajo de hasta tres estudiantes, que tenía como objetivo aplicar el Paradigma Orientado a Objetos (POO) y la utilización de estructuras de datos para dar solución a problemas típicos de las áreas de Calculo I, Matemáticas Discretas I y Física básica.

\section{Aprendizaje Basado en Proyectos}

Los orígenes del ABPrj se remontan a inicios del siglo XX con el nacimiento de la Escuela Progresista en Estados Unidos, cuando John Dewey argumentaría que los establecimientos educativos debían ser un escenario de creación de nuevas prácticas significativas para el desenvolvimiento del individuo en la sociedad, sobre la base del concepto de "aprender haciendo" con el denominado método del problema, en el cual los alumnos trabajan en equipo para resolver un problema a través del conocimiento multidisciplinar (CaeiroRodríguez, 2018). Igualmente, en 1918 William Heard Kilpatrick sentó las bases del ABPrj tal como se aplica en la actualidad, haciendo hincapié en el rol activo del estudiante (Cascales y Carrillo, 2018), y estableciendo que esta metodología era la mejor forma de impulsar las capacidades innatas del estudiante motivándolos al aprendizaje (GarcíaVarcálcel y Gómez-Pablos, 2017).

El ABPrj establece una responsabilidad de trabajo que está alineado con los indicadores de aprendizaje y las capacidades establecidas en los syllabus de las asignaturas (Toledo y Sánchez, 2018), además de usar un enfoque constructiva que involucra el entorno, fomenta las habilidades individuales del estudiante y la utilización de los saberes alcanzados a lo largo de su vida académica, con la meta de prepararlos para la inserción laboral (Mujica, 2012). Dentro de las principales características del ABPrj que afectan al estudiante se pueden mencionar que: 1) incentiva su auto-aprendizaje y la investigación, 2) le inculca el trabajo en equipo, 3) se involucra en la solución de problemas reales, 4) debe elaborar un producto final, 5) lo reta a desarrollar habilidades más avanzadas, y 6) le permite usar lo aprendido para solucionar nuevas cuestiones (Medina-Nicolalde y Tapia-Calvopiña, 2017). 
Por su parte, el docente adquiere un papel fundamental en la aplicación del ABPrj puesto que: 1) debe diseñar proyectos interesantes y que sean realizables según el nivel académico del estudiante, 2) es un motivador y guía del proceso, 3) aconseja a los estudiantes en cuanto a las técnicas a usar, 4) debe estar disponible ante cualquier duda o situación que sobrepase el conocimiento del estudiante, y 5) evalúa y retroalimenta de forma periódica lo realizado por los estudiantes (Manresa, Gil y Berbegal, 2020).

La metodología ABPrj es principalmente usada en la educación universitaria, en asignaturas con una orientación teórica-practica y que tienen un componente de formación profesional, es decir, que lo aprendido tiene una alta probabilidad de ser aplicado fuera del ambiente académico (Fajardo y Gil, 2019). En consecuencia, el estudiante aumenta el rendimiento y sus competencias al conseguir una representación más extensa de lo que le espera como profesional (Calvopiña, Medina y Tapia, 2017).

Para utilizar esta metodología, primero se establece un proyecto, que en algunos casos es definido por los mismos estudiantes que identifican el entorno, investigan sobre el tema, recolectan y analizan información relevante, planifican la gestión del proyecto, y diseñan e implementan prototipos que van solucionando problemas específicos, que en últimas se convierten en el producto final (Rodríguez, Vargas y Luna, 2010).

\section{EI ABPrj aplicado a la Ingeniería de Sistemas}

Cuando se trata de asignaturas cuyo objetivo es brindar bases teóricas que fundamenten la práctica universitaria y profesional del estudiante, sin importar el nivel académico, el ABPrj es una de las mejores herramientas pedagógicas a disposición de los docentes. Lo anterior se ve reflejado en investigaciones llevadas a cabo en diferentes cursos de los programas de Ingeniería de Sistemas de varias IES nacionales e internacionales donde se implementó el ABPrj, y cuyos resultados fueron satisfactorios. Por ejemplo; en la materia de Fundamentos de Programación de primer semestre se adaptó el ABPrj para desarrollar dos tipos de proyecto que dependían del nivel académico. El primero orientado a la implementación de juegos usando la herramienta Pseint, y el segundo para resolver problemas cotidianos mediante el uso de conceptos y modelamiento matemático (Parra, Amariles y Castro, 2016).

En Ingeniería de Software, se combinó el ABPrj con metodologías de desarrollo ágil como Scrum y Kanban para el diseño de situaciones reales, donde el estudiante debía construir un producto final, con base en los requerimientos del usuario (Sánchez y Blanco, 2012; Martín, 2020). También, el ABPrj se aplicó como herramienta para la gestión de una empresa escolar dedicada al desarrollo de software, con el objetivo fomentar en los integrantes del grupo de trabajo competencias relacionadas con la Ingeniería de Software y la habilidad para resolver problemas reales, lo cual incentivo el trabajo en equipo, el aprendizaje colaborativo e individual, habilidades de comunicación y negociación, liderazgo y la responsabilidad ética y social (Astorga, Flores, Ibarra, Mariscal, y Vizcarra, 2017). En Evaluación de Proyectos de Software se utilizaron técnicas de trabajo propias de la 
Ingeniería de Software con el ABPrj, con la meta de certificar la calidad de lo implementado a nivel empresarial (Villalobos-Abarca et al, 2018). En la asignatura de Base de Datos esta metodología ayudó al docente a desarrollar un proyecto que cubrió todas las etapas de la construcción de una aplicación, hasta la implementación conceptual del modelo de base de datos (Rico, Garay y Ruiz, 2018). En un curso de Computación Gráfica, el ABPrj se aplicó desde el 2004 hasta el 2007 con 150 alumnos que calificaron la metodología como positiva y motivadora (Martí et al, 2009). Y en Telecomunicaciones el ABPrj ayudó a los alumnos a disminuir el camino entre lo teórico y lo práctico, ya que en el proceso de aprendizaje se afrontaron situaciones de la vida real con cierta dificultad (González et al, 2014).

\section{Método}

La investigación se realizó bajo un enfoque descriptivo-cuantitativo, con el objetivo de evaluar la perspectiva que tuvieron los estudiantes al aplicar el ABPrj como herramienta pedagógica en el curso de IPOO. El enfoque descriptivo conceptualizo el tema permitiendo evidenciar la importancia del ABPrj desde el punto de vista pedagógico y de formación de competencias en futuros egresados. El enfoque cuantitativo permitió medir los datos recolectados al aplicar la encuesta diseñada, y que posteriormente fueron depurados y analizados para lograr las conclusiones de la temática estudiada.

Básicamente la investigación se llevó a cabo cinco fases: 1) Recolección de información, compuesta por la consulta de artículos científicos bajo palabras clave como: "ABPrj", "aprendizaje basado en proyectos", "aprendizaje activo" y "metodología constructivista", en bases de datos bibliográficas como Scopus, Web of Science, Elsevier y Redalyc, 2) Construcción del marco teórico, 3) Diseño y aplicación de la encuesta, 4) Análisis de datos recolectados, y 5) Conclusiones.

\section{Población}

La población objeto de estudio está representada por 73 estudiantes en total, 34 pertenecen al programa de TSI y 39 a IS. De los 73 estudiantes, 68 participaron de la muestra, 31 de Tecnología y 37 de Ingeniería, donde en general fueron 59 hombres (87\%) y 9 mujeres (13\%), con un promedio de edad de 20 años. Para comprobar que la muestra era representativa estadísticamente para el levantamiento de datos, se usó la fórmula 1 que permite establecer el mínimo de la población que debe participar (Villavicencio, 2017). La fórmula arrojó $M=63$, con lo que se puede concluir que la cantidad de estudiantes encuestados es representativa para la investigación. 
Donde:

$$
M=\frac{\frac{z^{2} \times p(1-p)}{e^{2}}}{1+\left(\frac{z^{2} \times(1-p)}{e^{2} N}\right)}
$$

$N$ (tamaño de población $)=73$

$e$ (margen de error) $=5 \%$

$p$ (nivel de confianza deseado $)=95 \%$

$z$ (cantidad de desviaciones estándar) que según $p$ debe ser $=1.96$

\section{Caracterización del curso}

El curso de IPOO está incluido en los pensum de los programas de TSI e IS, en ambos para segundo semestre. La información de la asignatura se consolida en la tabla 1.

\section{Tabla 1.}

Resumen de la asignatura

\begin{tabular}{|c|l|}
\hline Código: & $\mathbf{7 5 0 0 8 1 M}$ \\
\hline Nombre: & Introducción a la Programación Orientada a Objetos \\
\hline Créditos: & 4 \\
\hline Intensidad horaria: & 4 \\
\hline Habilitable: & $\mathrm{No}$ \\
\hline Validable: & $\mathrm{Si}$ \\
\hline Tipo: & Asignatura Básica Obligatoria \\
\hline Prerrequisito: & $750082 M$ Introducción a la Tecnología Informática \\
\hline
\end{tabular}

Fuente: Elaboración propia con base en EISC-IS (s.f) y EISC-TSI (s.f.)

A nivel general el objetivo de la asignatura es sentar las bases fundamentales que le permitan al estudiante dar soluciones a problemas usando la lógica algorítmica, y posteriormente implementar tales soluciones usando un lenguaje de programación que utilice el POO, además de las estructuras de datos típicas como arreglos, matrices, listas, pilas y colas, entre otras.

Es de resaltar, que la asignatura es pieza fundamental dentro de la malla curricular para ambos programas académicos, puesto que en adelante es prerrequisito para aproximadamente 3 asignaturas más de diferentes semestres. Asimismo, establece los conceptos acerca de la programación de computadores, necesarios para afrontar todo el ciclo de fundamentación y profesionalización del futuro egresado.

\section{Presentación del proyecto}

El proyecto fue entregado y socializado a los estudiantes desde la primera semana de clase. Se diseñó con la intención de ser interdisciplinar, basado en la realización de una aplicación computacional con enfoque matemático, que abordó contenidos curriculares de las asignaturas de: Cálculo I, Matemáticas Discretas I y Física básica. En este sentido, el docente proponente del proyecto se basó en los contenidos curriculares de las demás áreas 
para establecer los requerimientos de la aplicación a desarrollar, de tal manera que el alcance del proyecto fuera acorde con los conocimientos adquiridos hasta el momento, no fuera demasiado complejo y estuviera alineado con los objetivos de todas las asignaturas involucradas.

A lo largo del semestre académico se realizaron asesorías, con el objetivo de dar seguimiento, resolver inconvenientes y ayudar en cuestiones técnicas propias de la programación, puesto que para los estudiantes fue la primera experiencia al utilizar un lenguaje de programación bajo un paradigma estructurado, imperativo y orientado a objetos. La tabla 2 resume el enunciado.

\section{Tabla 2.}

Resumen del enunciado del proyecto

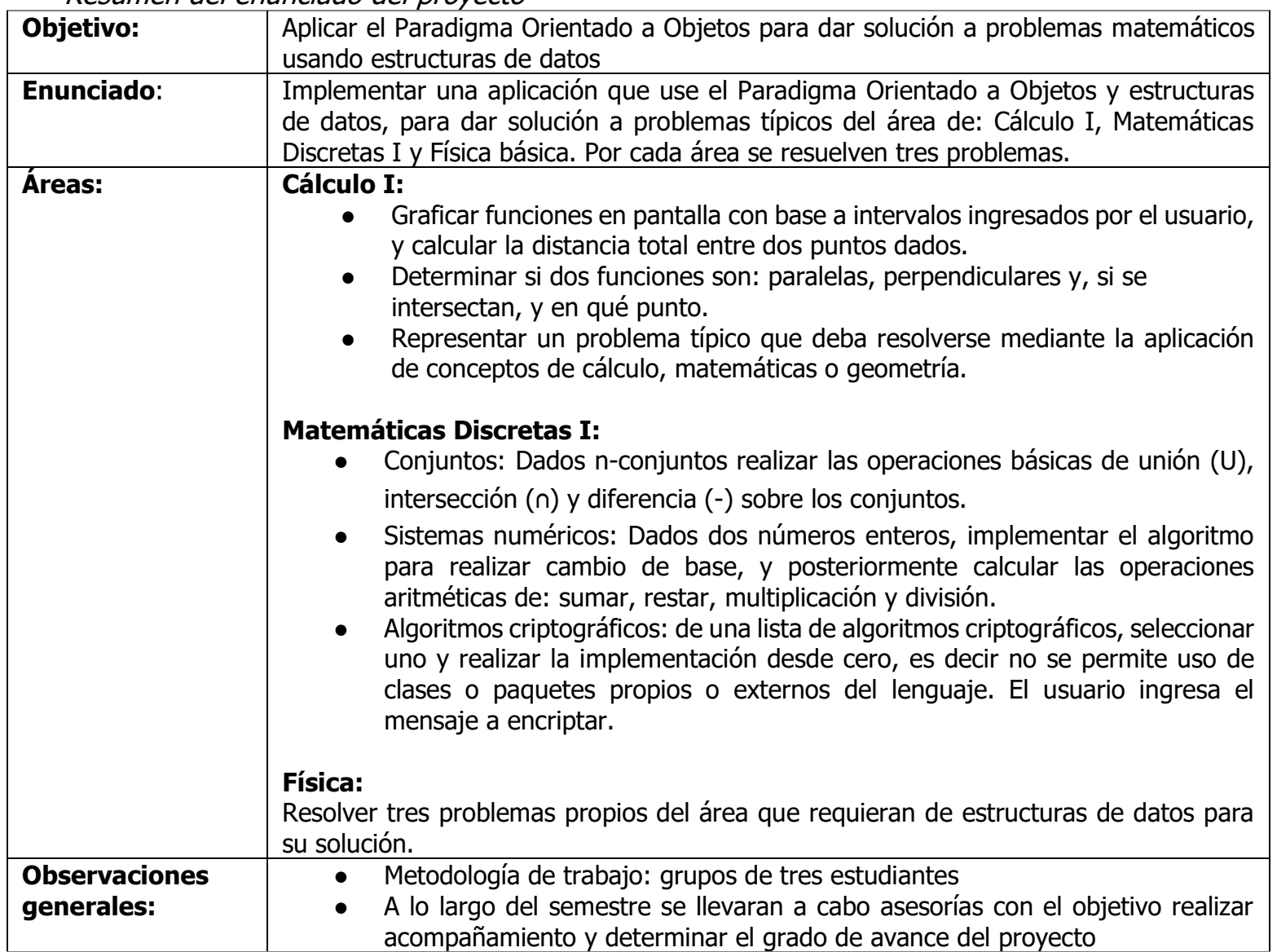

Fuente: Elaboración propia (2021)

\section{Diseño de la encuesta}

Para la recolección de datos se usó la herramienta de la encuesta, la cual fue aplicada de forma virtual por medio de Google Forms a través de un enlace enviado a los correos de los estudiantes. En el instrumento se utilizaron preguntas cerradas de selección de rango 
numérico, con la intención de facilitar las tareas de recopilación, depuración y análisis de datos.

El instrumento fue diseñado con base en el Cuestionario de Efectividad del Uso de Metodologías de Participación Activa (CEMPA) de Carrasco et al, (2015) que evaluó la percepción de la efectividad del uso del ABPrj en un curso de Contabilidad, y que fue validado por medio de técnicas estadísticas.

La encuesta aplicada en el presente estudio consta de 25 preguntas, 3 de ellas indagaron el género, la edad y la sede (Buga o Tuluá). Las otras 22 son de tipo escala numérica de 1 a 5 , donde 1 es la nota más baja y 5 la más alta cada uno de factores. Asimismo, estas 22 preguntas están distribuidas en las tres siguientes secciones:

1. Presentación del proyecto (4 preguntas): se pretendió evaluar todo lo relacionado con el enunciado del problema, sus objetivos, metodología, tiempos y resultado esperado, por medio de interrogantes relacionados con: la evaluación de los objetivos del proyecto, la relación y aplicabilidad de los contenidos curriculares del programa académico, y la descripción del producto final.

2. Desarrollo de competencias específicas (4 preguntas): orientada a determinar si la metodología usada logró incentivar todas aquellas habilidades, conocimientos y destrezas necesarias para llevar a cabo de forma apropiada una labor o trabajo. Para ello se indagó acerca de: la evaluación de la aplicabilidad de los conceptos adquiridos en la asignatura, relación entre conceptos teóricos y la práctica, el aumento del interés por la programación de computadoras y aporte a su formación profesional.

3. Desarrollo de competencias generales:

- Instrumentales (4 preguntas): se buscó evaluar que tanto la metodología permitió desarrollar e incluir destrezas cognoscitivas, capacidad de aprendizaje, y el uso de habilidades técnicas, a través de interrogantes como: obtención de habilidades para gestión del proyecto, para la resolución de problemas y la investigación.

- Sistémicas (5 preguntas): enfocada a determinar el grado de influencia de la metodología del proyecto en el desarrollo individual del estudiante en cuanto a las competencias relacionadas con la realización de tareas por objetivos, aumento de la creatividad y delegación de funciones a los miembros del equipo de trabajo.

- Interpersonales (5 preguntas): dirigida a establecer la importancia de la metodología en el desarrollo individual en cuanto a las competencias sociales relacionadas con: el incentivo por la comunicación, la automotivación, trabajo en equipo y desarrollo del liderazgo.

Cabe mencionar que la encuesta fue totalmente anónima, con el propósito de que el alumno se sintiera más cómodo al responder, y expresar con libertad su punto de vista, 
siendo un factor fundamental de retroalimentación para futuras investigaciones. La encuesta se puede ver en el Anexo I.

\section{Resultados}

En esta sección, primero se procederá a visualizar y analizar los datos obtenidos por cada una de las tres secciones en que se dividió la herramienta: 1) Presentación del proyecto, 2) Desarrollo de competencias específicas y 3) Desarrollo de competencias generales.

En las tablas 3, 4 y 6 se omitió la calificación 1 y 2 (las de menor peso) al no estar presentes. Las tablas 5 y 7 excluyeron la calificación 1.

\section{Presentación del proyecto}

En la evaluación de la Presentación del proyecto los resultados evidencian que este elemento del ABPrj, el cual es uno de los más importantes, ya que sienta las bases para las etapas posteriores, cumplió con el objetivo de describir el propósito y metas del mismo, al tener una media general de 4.6 pts., sobre 5.0, que representa el 92\% de aprobación por parte de los estudiantes. Lo anterior también se refleja en el resultado de la moda estadística sobre la calificación, cuyo valor fue de $M=5.0$ pts., seguida de $M=4.0$ pts.

Tabla 3.

Resumen calificaciones sección Presentación del proyecto

\begin{tabular}{|c|c|c|c|c|c|c|c|c|}
\hline \multirow{2}{*}{ \# } & \multirow{2}{*}{ Pregunta } & \multicolumn{3}{|c|}{ Calificación General } & \multirow{2}{*}{$\begin{array}{c}\text { Media } \\
\text { Tecnologí } \\
\mathbf{a} \\
\end{array}$} & \multirow{2}{*}{$\begin{array}{c}\text { Media } \\
\text { Ingenierí } \\
\mathbf{a} \\
\end{array}$} & \multirow{2}{*}{$\begin{array}{c}\text { Media } \\
\text { Gener } \\
\text { al }\end{array}$} & \multirow{2}{*}{$\begin{array}{c}\text { \% } \\
\text { Gener } \\
\text { al } \\
\end{array}$} \\
\hline & & 3 & 4 & 5 & & & & \\
\hline 1 & $\begin{array}{l}\text { Objetivo del proyecto } \\
\text { claramente definido }\end{array}$ & 4 & 23 & 41 & 4,6 & 4,5 & 4,6 & 91 \\
\hline 2 & $\begin{array}{l}\text { Objetivo del proyecto } \\
\text { relacionado con el } \\
\text { contenido curricular }\end{array}$ & 2 & 14 & 52 & 4,8 & 4,7 & 4,8 & 95 \\
\hline 3 & $\begin{array}{l}\text { Objetivo del proyecto } \\
\text { permitió aplicar conceptos } \\
\text { de otras áreas del } \\
\text { conocimiento }\end{array}$ & 3 & 30 & 35 & 4,4 & 4,5 & 4,5 & 89 \\
\hline 4 & $\begin{array}{l}\text { El proyecto describe cuál } \\
\text { debe ser el producto final }\end{array}$ & 1 & 21 & 46 & 4,8 & 4,6 & 4,7 & 94 \\
\hline
\end{tabular}

Fuente: Elaboración propia (2021)

Cabe resaltar que a juicio del investigador se debe replantear para futuros proyectos la metodología que hace referencia a la pregunta 3, relacionada a si el objetivo del proyecto permitió al estudiante aplicar los conceptos de otras asignaturas y áreas del conocimiento, pues su media fue la más baja con 4.5 pts., y un porcentaje del $89 \%$ de aprobación. Esto a 
pesar de que se tuvieron en cuenta los contenidos curriculares de otras asignaturas del pensum en el semestre, y que los conocimientos adquiridos con anterioridad (semestre anterior o educación secundaria) fueran los suficientes para resolver los problemas.

\section{Desarrollo de competencias específicas}

En cuanto a la evaluación de la sección de Desarrollo de competencias específicas, los resultados a criterio del investigador fueron muy sobresalientes, al lograrse una calificación promedio de 4.7 pts., que equivalen al 93\% de aprobación. Máxime, en la pregunta número 5 , que obtuvo un promedio de 4.9 , lo cual es muy significativo, ya que, a juicio de los estudiantes, fue altamente necesario aplicar los conceptos obtenidos en la asignatura, que era uno de los objetivos más importantes del proyecto y la utilización del ABPrj.

Tabla 4.

Resumen calificaciones sección Desarrollo de competencias específicas

\begin{tabular}{|c|c|c|c|c|c|c|c|c|}
\hline \multirow{2}{*}{ \# } & \multirow{2}{*}{ Pregunta } & \multicolumn{3}{|c|}{ Calificación General } & \multirow{2}{*}{$\begin{array}{l}\text { Media } \\
\text { Tecnologí } \\
\text { a }\end{array}$} & \multirow{2}{*}{$\begin{array}{l}\text { Media } \\
\text { Ingenierí } \\
\text { a }\end{array}$} & \multirow{2}{*}{$\begin{array}{l}\text { Media } \\
\text { Gener } \\
\text { al }\end{array}$} & \multirow{2}{*}{$\begin{array}{l}\% \\
\text { Gener } \\
\text { al }\end{array}$} \\
\hline & & 3 & 4 & 5 & & & & \\
\hline 5 & $\begin{array}{l}\text { Aplicación de conceptos } \\
\text { adquiridos en la asignatura } \\
\text { de IPOO }\end{array}$ & 1 & 11 & 56 & 4,9 & 4,8 & 4,9 & 97 \\
\hline 6 & $\begin{array}{l}\text { Relación y aplicación entre } \\
\text { lo teórico y la práctica }\end{array}$ & 2 & 20 & 46 & 4,6 & 4,6 & 4,6 & 92 \\
\hline 7 & $\begin{array}{l}\text { Aumento del interés por la } \\
\text { asignatura y la } \\
\text { programación }\end{array}$ & 7 & 18 & 43 & 4,6 & 4,5 & 4,6 & 91 \\
\hline 8 & $\begin{array}{l}\text { Aporte a la formación } \\
\text { profesional }\end{array}$ & 3 & 21 & 44 & 4,7 & 4,5 & 4,6 & 92 \\
\hline
\end{tabular}

Fuente: Elaboración propia (2021)

De nuevo, es importante resaltar los resultados de la pregunta 8 , que fue la segunda con mejor porcentaje de aprobación. Esto es significativo, si se tiene en cuenta que los estudiantes participantes en la investigación pertenecen a segundo semestre, lo que significa que posiblemente aún no tienen una visión muy amplia y clara de su papel como profesionales. En este caso, los resultados avalan la utilización del ABPrj como pieza fundamental en el desarrollo de conciencia respecto a la formación y perfil laboral.

\section{Desarrollo de competencias generales}

\subsection{Instrumentales}

Para la evaluación del desarrollo de competencias instrumentales se obtuvo un promedio general de calificación de 4.4 pts., representando un $88 \%$ de aprobación, y una moda $\mathrm{M}=$ 5.0 . 
Tabla 5.

Resumen calificaciones sección Desarrollo de competencias generales - Instrumentales

\begin{tabular}{|c|c|c|c|c|c|c|c|c|c|}
\hline \multirow{2}{*}{ \# } & \multirow{2}{*}{ Pregunta } & \multicolumn{4}{|c|}{ Calificación General } & \multirow{2}{*}{$\begin{array}{c}\text { Media } \\
\text { Tecnologí } \\
\text { a }\end{array}$} & \multirow{2}{*}{$\begin{array}{c}\text { Media } \\
\text { Ingenierí } \\
\text { a }\end{array}$} & \multirow{2}{*}{$\begin{array}{l}\text { Media } \\
\text { Genera } \\
\quad \text { I }\end{array}$} & \multirow{2}{*}{$\begin{array}{c}\% \\
\text { Gener } \\
\text { al }\end{array}$} \\
\hline & & 2 & 3 & 4 & 5 & & & & \\
\hline 9 & $\begin{array}{l}\text { Logro de habilidades } \\
\text { para la organización y } \\
\text { planificación del tiempo }\end{array}$ & 2 & 6 & 30 & 30 & 4,3 & 4,3 & 4,3 & 86 \\
\hline $\begin{array}{l}1 \\
0\end{array}$ & $\begin{array}{l}\text { Logro de habilidades } \\
\text { para la resolución de } \\
\text { problemas }\end{array}$ & 0 & 3 & 30 & 35 & 4,5 & 4,4 & 4,5 & 89 \\
\hline $\begin{array}{l}1 \\
1\end{array}$ & $\begin{array}{l}\text { Logro de habilidades } \\
\text { para la toma de } \\
\text { decisiones }\end{array}$ & 0 & 5 & 29 & 34 & 4,5 & 4,4 & 4,5 & 89 \\
\hline $\begin{array}{l}1 \\
2\end{array}$ & $\begin{array}{l}\text { Aplicación de temas } \\
\text { investigados en otras } \\
\text { asignaturas y áreas del } \\
\text { conocimiento }\end{array}$ & 0 & 5 & 28 & 35 & 4,5 & 4,3 & 4,4 & 88 \\
\hline
\end{tabular}

Fuente: Elaboración propia (2021)

Es importante resaltar que esta sección fue la de menor calificación, siendo los ítems 9 y 12 los de menor promedio, pero que igual son resultados muy satisfactorios. En este caso, para futuras implementaciones del ABPrj, el docente debe planear y utilizar estrategias que permitan dar a conocer al estudiante, técnicas para el establecimiento de un cronograma de actividades y tiempos como los diagramas de Gantt, que le ayuden de cierta manera a lograr mejores competencias y habilidades en este criterio.

\subsection{Sistémicas}

En la evaluación de la subsección de desarrollo de competencias sistémicas, los resultados también fueron muy sobresalientes, al obtener una calificación promedio de 4.5 pts., que equivalen al $90 \%$ de aprobación. Como hecho relevante es importe resaltar los resultados de las preguntas 13 y 16, siendo las de mayor porcentaje, y en las cuales se logró, a criterio del investigador dos objetivos fundamentales del ABPrj como lo son el incentivar la creatividad y la investigación en el estudiante como factores decisivos de la formación activa y el logro de competencias profesionales (Queiruga, Sáiz y Montero, 2019).

Tabla 6.

Resumen calificaciones sección Desarrollo de competencias generales - Sistémicas

\begin{tabular}{|c|c|c|c|c|c|c|c|c|}
\hline \multirow[t]{2}{*}{ \# } & \multirow[t]{2}{*}{ Pregunta } & \multicolumn{3}{|c|}{$\begin{array}{c}\text { Calificación } \\
\text { General }\end{array}$} & \multirow{2}{*}{$\begin{array}{c}\text { Media } \\
\text { Tecnologí } \\
\mathbf{a}\end{array}$} & \multirow{2}{*}{$\begin{array}{c}\text { Media } \\
\text { Ingenierí } \\
\mathbf{a}\end{array}$} & \multirow{2}{*}{$\begin{array}{c}\text { Media } \\
\text { Gener } \\
\text { al }\end{array}$} & \multirow{2}{*}{$\begin{array}{c}\% \\
\text { Gener } \\
\text { al }\end{array}$} \\
\hline & & 3 & 4 & 5 & & & & \\
\hline $\begin{array}{l}1 \\
3\end{array}$ & Incentivo de la creatividad & 6 & 26 & 36 & 4,6 & 4,5 & 4,6 & 91 \\
\hline $\begin{array}{l}1 \\
4\end{array}$ & $\begin{array}{l}\text { Establecimiento de tareas } \\
\text { por objetivo }\end{array}$ & 5 & 27 & 36 & 4,5 & 4,5 & 4,5 & 90 \\
\hline $\begin{array}{l}1 \\
5\end{array}$ & $\begin{array}{l}\text { Establecimiento de un plan } \\
\text { de actividades }\end{array}$ & 6 & 30 & 32 & 4,4 & 4,5 & 4,5 & 89 \\
\hline
\end{tabular}




\begin{tabular}{|c|l|c|c|c|c|c|c|c|}
1 & Incentivo hacia la & 2 & 23 & 43 & 4,7 & 4,6 & 4,7 & 93 \\
\hline $\begin{array}{l}\text { investigación } \\
\mathbf{7}\end{array}$ & Delegación de tareas & 8 & 32 & 28 & 4,3 & 4,4 & 4,4 & 87 \\
\hline
\end{tabular}

Fuente: Elaboración propia (2021)

Igualmente, con el objetivo de que la metodología del ABPrj sea más efectiva y se logre mayor impacto en aquellas competencias llamadas gerenciales como las evaluadas en la pregunta 15 y 17, el docente debe construir estrategias que ayuden al estudiante en la planificación de tareas por medio de la aplicación de la Ingeniería de Software, ya que esta actividad se considera como uno de los componentes que más contribuyen a garantizar el éxito de un proyecto, al tener en cuenta aspectos como: el objetivo de proyecto, el tiempo disponible, recursos necesarios para su consecución y manejo de personal (Pressman, 1988).

\subsection{Interpersonales}

En la evaluación de la subsección de desarrollo de competencias interpersonales, los resultados fueron destacados, al obtener una calificación promedio de 4.4 pts., que equivalen al $88 \%$ de aprobación. Al igual que en las evaluaciones anteriores, en esta subsección de la encuesta también la moda en la calificación fue de $M=5.0$ pts. De resaltar el ítem 18, donde la estrategia del ABPrj implementada por el docente trato de incentivar a los estudiantes por aprender y no solo para aprobar la asignatura por medio de una motivación intrínseca, en la que se plantearon actividades desafiantes que buscaban un mayor compromiso, aplicación de lo aprendido y lo investigado.

Tabla 7.

Resumen calificaciones sección Desarrollo de competencias generales - Interpersonales

\begin{tabular}{|c|c|c|c|c|c|c|c|c|c|}
\hline \multirow[t]{2}{*}{ \# } & \multirow{2}{*}{ Pregunta } & \multicolumn{4}{|c|}{$\begin{array}{l}\text { Calificación } \\
\text { General }\end{array}$} & \multirow{2}{*}{$\begin{array}{c}\text { Media } \\
\text { Tecnologí } \\
\text { a }\end{array}$} & \multirow{2}{*}{$\begin{array}{c}\text { Media } \\
\text { Ingenierí } \\
\text { a }\end{array}$} & \multirow{2}{*}{$\begin{array}{c}\text { Media } \\
\text { Genera } \\
\text { I }\end{array}$} & \multirow{2}{*}{$\begin{array}{c}\% \\
\text { Genera } \\
\text { I }\end{array}$} \\
\hline & & 2 & 3 & 4 & 5 & & & & \\
\hline $\begin{array}{l}1 \\
8\end{array}$ & $\begin{array}{l}\text { Incentivo de la } \\
\text { automotivación }\end{array}$ & 1 & 8 & 25 & 34 & 4,6 & 4,5 & 4,6 & 91 \\
\hline $\begin{array}{l}1 \\
9\end{array}$ & $\begin{array}{l}\text { Incentivo por las } \\
\text { relaciones y la } \\
\text { comunicación } \\
\text { interpersonal }\end{array}$ & 4 & 8 & 28 & 28 & 4,3 & 4,2 & 4,3 & 85 \\
\hline $\begin{array}{l}2 \\
0\end{array}$ & $\begin{array}{l}\text { Incentivo por el trabajo } \\
\text { en equipo }\end{array}$ & 1 & 6 & 29 & 32 & 4,5 & 4,5 & 4,5 & 90 \\
\hline $\begin{array}{l}2 \\
1\end{array}$ & $\begin{array}{l}\text { Adquisición de } \\
\text { habilidades para la } \\
\text { resolución de conflictos } \\
\text { en el equipo de trabajo }\end{array}$ & 2 & 7 & 28 & 31 & 4,4 & 4,4 & 4,4 & 88 \\
\hline $\begin{array}{l}2 \\
2\end{array}$ & Aplicación de liderazgo & 1 & 11 & 23 & 33 & 4,4 & 4,4 & 4,4 & 88 \\
\hline
\end{tabular}

Fuente: Elaboración propia (2021) 
Al igual que en la subsección de competencias sistémicas, los ítems 19, 21 y 22, relacionados con aspectos de tipo gerencial y gestión del trabajo fueron los de menor media general. En este aspecto, el desarrollo de habilidades para el trabajo en equipo no es algo trivial, ya que es común en algunos alumnos la preferencia por el trabajo individual, por ejemplo, por la sensación de carencia de talento en sus compañeros, o pensar que se avanza más rápido solo con sus propias ideas. Es aquí, donde el aprendizaje colaborativo puede ayudar a cambiar creencias que limitan el desarrollo de habilidades de comunicación y trabajo grupal (Jaramillo-Valencia, y Quintero-Arrubla, 2021).

Nuevamente, se hace necesario replantear las estrategias utilizadas para incentivar estas competencias, por medio de lecturas y actividades prácticas donde el estudiante pueda simular escenarios que requieran de la aplicación de conceptos administrativos, por ejemplo, con el uso de un marco de trabajo ágil para construir software como eXtreme Programming (XP) o SCRUM, recomendadas para proyectos de corta duración.

\subsection{Prueba $t$ de Student}

Después de obtener los datos consolidados de las 22 preguntas de la encuesta diseñada, donde el promedio general fue de 4.5, se pudo establecer que a simple vista no hubo mayores diferencias en los resultados de la evaluación entre los estudiantes de Tecnología y los de Ingeniería. Algo que de cierta manera se esperaba, ya que en ambos programas la asignatura se dicta en segundo semestre y tiene similar prerrequisito, es decir hasta el momento los estudiantes cuentan con el mismo conocimiento y preparación en todas las asignaturas relacionadas con el proyecto. La figura 1 muestra el comparativo de promedios para las 22 preguntas.

\section{Figura 1.}

Comparativo de promedio de calificaciones para las 22 preguntas

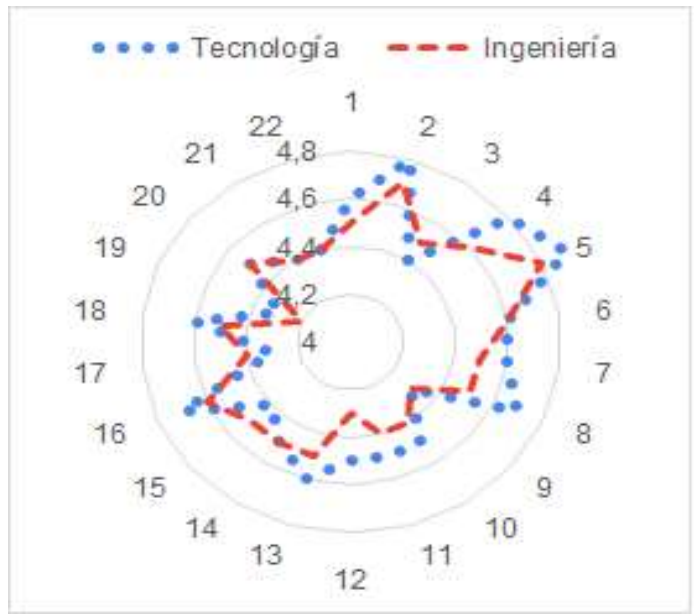

Fuente: Elaboración propia (2021) 
Sin embargo, se aplicó la prueba $t$ de Student con el objetivo de verificar si realmente existieron diferencias significativas entre los resultados obtenidos por los estudiantes de ambos programas.

Para aplicar $t$ de Student primero se usó la prueba $F$ para determinar si las varianzas eran iguales. Para $F$ se determinó un $95 \%$ de nivel de confianza y un $5 \%$ de significancia en ambos grupos, bajo las hipótesis:

- $H O$ (Hipótesis nula): Las varianzas son iguales para ambos grupos

- H1 (Hipótesis alternativa): Las varianzas son diferentes para ambos grupos

Y teniendo como criterios:

- HO se rechaza si $P \leq$ nivel de significancia

- $H O$ se acepta si $P>$ nivel de significancia

Al aplicar la prueba $F$ arrojó que $P=0.29489$

Como $P(0.29489)>$ nivel de significancia (0.05), se acepta $H O$.

Por lo tanto, se aplicó la Prueba $t$ para dos colas que suponen varianzas semejantes para ambos grupos.

Bajo las hipótesis de:

- HO (Hipótesis nula): No existe diferencia estadísticamente significativa en el promedio de calificaciones de ambos grupos

- $H 1$ (Hipótesis alternativa): Si existe diferencia estadísticamente significativa en el promedio de calificaciones de ambos grupos

Y teniendo como criterios:

- $H 0$ se rechaza si $P \leq$ nivel de significancia

- HO se acepta si $P>$ nivel de significancia

Los resultados fueron los siguientes: El valor Estadístico $t=1.292330317$, y $P=$ 0.203308

Como P (0.203308) > nivel de significancia (0.05), se acepta HO. Por lo tanto, se determina que no existe diferencia estadísticamente significativa en el promedio de calificaciones de ambos grupos, confirmando lo argumentado con anterioridad.

También fue de interés conocer si la edad fue un factor significativo en los datos obtenidos de la herramienta, en la cual la media de edad de los estudiantes de Tecnología fue de 19 años y un promedio de calificación de 4.54 pts. Mientras en Ingeniería fue de 20 
años, y 4.41 pts., respectivamente. La figura 2 muestra el gráfico de dispersión entre las edades y el promedio de calificación para ambos grupos.

\section{Figura 2.}

Gráfico de dispersión entre las edades y el promedio de calificación

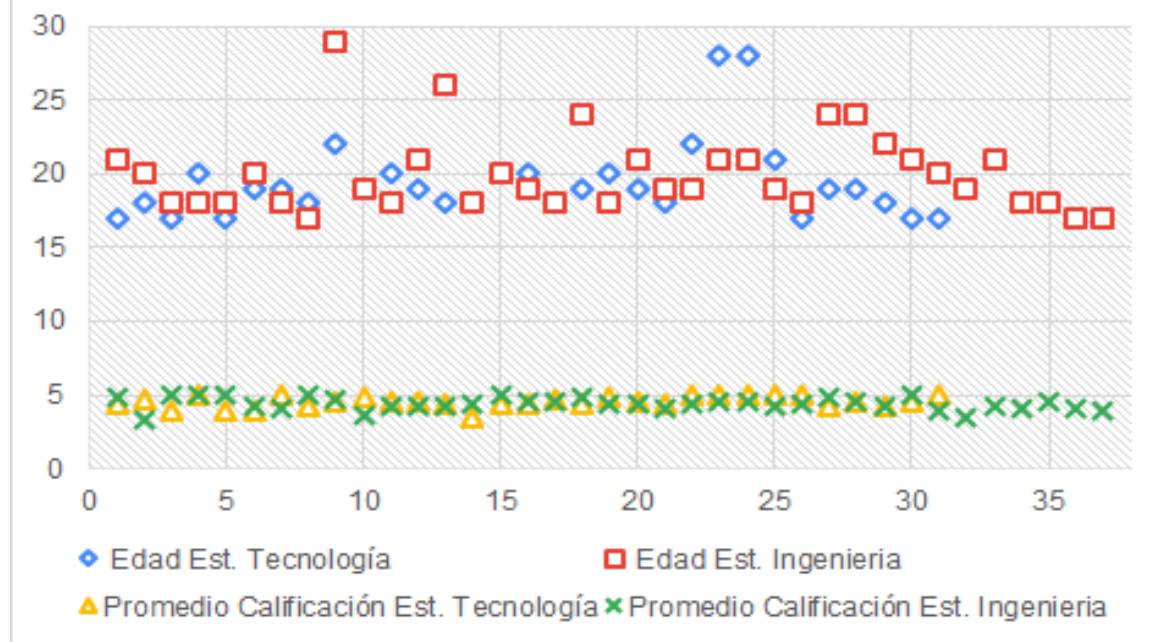

Fuente: Elaboración propia

Para establecer si la edad de los estudiantes de ambos programas fue un factor significativo que influyo en las respuestas de la encuesta, se aplicó el coeficiente de Pearson $(r)$, y el coeficiente de Determinación $\left(R^{2}\right)$ para interpretar $r$. Los resultados por programa fueron los siguientes:

- Tecnología: $r=0.40760$. Como $0<r<1$, se puede determinar que si bien es cierto existe una correlación positiva, es decir si hay correlación entre la edad y el promedio de la evaluación, esta es medianamente débil, y que según $R^{2}$ es del $16.61 \%$.

- Ingeniería: $r=0.19436$. Como $0<r<1$, se puede determinar que si bien es cierto existe una correlación positiva, es decir si hay correlación entre la edad y el promedio de la evaluación, esta es muy débil, y que según $R^{2}$ es de tal solo el $3.78 \%$.

\section{Discusiones y conclusiones}

El ABPrj ha demostrado ser una herramienta pedagógica de gran valor, independientemente del nivel educativo donde se implemente. En este caso, los resultados de la actual investigación son muy prometedores, si se tiene en cuenta que la calificación promedio fue de 4.5 pts., lo que equivale al $90 \%$ de aprobación, sumado a que era la primera vez que los estudiantes se enfrentaban al desarrollo de un proyecto bajo el enfoque de la metodología. Por otra parte, las pruebas estadísticas confirmaron la hipótesis del investigador, en cuanto a que no existieron diferencias muy marcadas en los estudiantes de Tecnología e Ingeniería respecto a la evaluación de la estrategia implementada. Igualmente, aunque el promedio de edad fue casi igual para ambos grupos (19 y 20 respectivamente), 
se logró establecer que existió una correlación entre la edad y el promedio de la evaluación, siendo estas débiles, alcanzando su mayor diferencia en los estudiantes de Tecnología con un $16.61 \%$, pero que en términos generales se puede concluir que no se existió correlación entre las dos variables.

Por otra parte, es imperioso que las todas las instituciones de educación reformulen sus políticas educativas con el objetivo de actualizar sus programas educativos y currículos, y le brinden a los docentes oportunidades de estudios orientados a la utilización de nuevas metodologías de aprendizaje-enseñanza, así como a la inclusión de la herramientas tecnológicas, de tal forma que se alcance en los alumnos aprendizajes más activos y destrezas acordes a la sociedad digital, lo que trascienda en una mayor accesibilidad del egresado al mundo laboral.

Asimismo, la investigación lleva a repensar el nuevo papel del docente y las estrategias utilizadas por este en sus labores de enseñanza, las cuales deben involucrar más al estudiante, un actor que históricamente ha estado rezagado debido a currículos tradicionales en los cuales el docente era el actor principal, que diseñaba de forma autónoma los contenidos que para el eran los más acordes según su experiencia docente y laboral. En la actualidad el estudiante exige ser partícipe del diseño de los programas de estudio. Quiere que su proceso de aprendizaje este acorde con el perfil profesional que necesita la sociedad, y esto lo se logra en gran medida con la adopción de metodologías más activas, enfocadas a problemas reales y centradas en ellos.

Finalmente, se espera que los resultados del actual estudio sirvan de retroalimentación para el ejercicio docente y fuente para futuras iniciativas e investigaciones tendientes a implementar estrategias novedosas que ayuden al alumno en la formación y obtención de todas aquellas capacidades individuales y laborales requeridas por la sociedad y el entorno laboral actual.

\section{Referencias}

Angarita, M., Fernández, F. y Duarte, J. (2016). Formación de ingenieros interdisciplinarios a través de una metodología activa con temáticas integradoras. Saber, Ciencia Y Libertad, 11(2), 177-187. https://doi.org/10.18041/23823240/saber.2016v11n2.555

Astorga, M., Flores, B., Ibarra, J., Mariscal, J., y Vizcarra, L. (2017). Impacto del aprendizaje basado en proyectos implementado en una empresa escolar de Base Tecnológica dedicada al desarrollo de Software. ReCIBE, 4(4), IV. https://doi.org/10.32870/recibe.v4i4.54 
Barrera, M. (2017). Aprendizaje basado en proyectos colaborativos mediados por tic para el desarrollo de competencias en estadística. [Tesis de Maestría, Universidad $\begin{array}{lllll}\text { Pedagógica } & y & \text { Tecnológica Colombia]. }\end{array}$ https://repositorio.uptc.edu.co/bitstream/001/2325/1/TGT-966.pdf

Botella, A. y Ramos, P. (2019). Investigación-acción y aprendizaje basado en proyectos. Una revisión bibliográfica. Perfiles educativos, 41(163), 127-141. https://doi.org/10.22201/iisue.24486167e.2019.163.58923

Bravo, A., Arenas, J. y Pineda, E. (2019). El aprendizaje de la geometría con GeoGebra, un enfoque de aprendizaje por problemas. Revista Docencia Universitaria, 20(2), 55-67. https://revistas.uis.edu.co/index.php/revistadocencia/article/view/10522/10959

Cabedo, L. et al. (2017). La metodología de aprendizaje basado en proyectos (ABP) aplicada a asignaturas de ciencia de los materiales en ingeniería en la red IdM@ti. MaterialES, 1(2), 25-28. http://hdl.handle.net/10234/169913

Caeiro-Rodríguez, M. (2018) Aprendizaje Basado en la Creación y Educación Artística: proyectos de aula entre la metacognición y la metaemoción. Arte, Individuo y Sociedad, 30(1), 159-177. https://doi.org/10.5209/ARIS.57043

Calvopiña, M., Medina, M. y Tapia, M. (2017). El aprendizaje basado en proyectos desde una perspectiva interdisciplinar. Lecturas: Educación Física Y Deportes, 22(235), 1521. https://www.efdeportes.com/efdeportes/index.php/EFDeportes/article/view/33

Capote, G., Rizo, N. y Bravo, G. (2016). La formación de ingenieros en la actualidad. Una explicación necesaria. Revista Universidad y Sociedad, 8(1), 21-28. http://scielo.sld.cu/scielo.php?script=sci_arttext\&pid=S221836202016000100004\&l $\mathrm{ng}=\mathrm{es} \& \mathrm{t} \operatorname{lng}=\mathrm{es}$

Carrasco, A., et al. (2015). Diseño y validación de un cuestionario que mide la percepción de efectividad del uso de metodologías de participación activa (CEMPA). El caso del Aprendizaje Basado en Proyectos (ABPrj) en la docencia de la contabilidad. Innovar, 25(58), 143-158. http://dx.doi.org/10.15446/innovar.v25n58.52439

Cascales, A. y Carrillo, M. (2018). Aprendizaje basado en proyectos en educación infantil: cambio pedagógico y social. Revista Ibero-americana de Educação, (76), 79-98. https://doi.org/10.35362/rie7602861

CONPES 3809. (2014). Documento CONPES 3809. Departamento Nacional de Planeación. https://colaboracion.dnp.gov.co/CDT/Conpes/Econ\%C3\%B3micos/3809.pdf 
Correa, L. (2019). Aplicación de estrategias didácticas enfocadas en aprendizaje activo en ingeniería. Estudio de caso para un espacio académico de sistemas de control. Actualidades Pedagógicas, (74), 49-77. https://doi.org/10.19052/ap.vol1.iss74.3

Durán, C. y Rosado, A. (2020). Aprendizaje activo e innovación en estudiantes de ingeniería. Revista Colombiana de Tecnologías de Avanzada, 1(35), 127-135. http://revistas.unipamplona.edu.co/ojs_viceinves/index.php/RCTA/article/view/392

EISC. (2009). Proyecto Educativo del Programa de Ingeniería de Sistemas, Escuela de Ingeniería de Sistemas y Computación (EISC). https://drive.google.com/file/d/0B4MYckRwqozAVkEtbHA4ZmVDR3M/view

EISC-IS. (s.f). Escuela de Ingeniería de Sistemas y Computación, Ingeniería de Sistemas. Universidad del Valle. http://eisc.univalle.edu.co/index.php/programasacademicos/ingenieria-de-sistema

EISC-TSI. (s.f). Escuela de Ingeniería de Sistemas y Computación, Tecnología en Sistemas de Información. Universidad del Valle. http://eisc.univalle.edu.co/index.php/programas-academicos/tecnologia-ensistemas-de-informacion

Fajardo, E. y Gil, B. (2019). El aprendizaje basado en proyectos y su relación con el desarrollo de competencias asociadas al trabajo colaborativo. Revista Amauta, 17(33), pp.103-118. http://dx.doi.org/10.15648/am.33.2019.8

Fernández, F. y Duarte, J. (2013). El Aprendizaje basado en Problemas como Estrategia para el Desarrollo de Competencias Específicas en Estudiantes de Ingeniería. Formación Universitaria, 6(5) 29-38. http://dx.doi.org/10.4067/S071850062013000500005

Fernández-Cabezas, M. (2017). Aprendizaje basado en proyectos en el ámbito universitario: una experiencia de innovación metodológica en educación. Revista INFAD de Psicología. International Journal of Developmental and Educational Psychology, 2(1), 269-278. https://doi.org/10.17060/ijodaep.2017.n1.v2.939

García, C., Montaño, J. y Pérez, C. (2019). Aprendizaje basado en proyectos para el desarrollo comunitario, una experiencia en la formación de Ingenieros Civiles. Conrado, 15(68),

130-134. https://conrado.ucf.edu.cu/index.php/conrado/article/view/994/1016

García, J. y Pérez, J. (2018). Aprendizaje basado en proyectos: método para el diseño de actividades. CEF, (10), 37-63. https://tecnologia-cienciaeducacion.com/index.php/TCE/article/viewFile/194/176 
García, M., Gómez, A., Solano, N. y Fernández, R. (2020). Las creencias de los futuros maestros sobre la enseñanza y aprendizaje de las matemáticas. Revista Espacios, 41(9), 14. https://www.revistaespacios.com/a20v41n09/20410914.html

García-Varcálcel, A. y Gómez-Pablos, V. (2017). Aprendizaje basado en proyectos (ABP): evaluación desde la perspectiva de alumnos de Educación Primaria. Revista de Investigación Educativa, 35(1), 113-131. http://dx.doi.org/10.6018/rie.35.1.246811

González-Jorge, H., Roca, D., Torres, S., Armesto, J. y Puente, I. (2014). Una experiencia de Aprendizaje Basado en Proyectos en el ámbito tecnológico: Diseño de un sistema de navegación indoor de bajo coste. Revista de Formación e Innovación Educativa Universitaria, $\quad 7(1)$, 8-19. http://refiedu.webs.uvigo.es/Refiedu/Vol7_1/REFIEDU_7_1_2.pdf

Gürbüz, R. y Erdem, E. (2016). Relationship between mental computation and mathematical reasoning. Cogent Education, 3(1), 1-19. https://doi.org/10.1080/2331186X.2016.1212683

Jaramillo-Valencia, B., y Quintero-Arrubla, S. (2021). Trabajando en equipo: múltiples perspectivas acerca del trabajo cooperativo y colaborativo. Educación Y Humanismo, 23(41). https://doi.org/10.17081/eduhum.23.41.4188

Laguado, R., Ramírez, R. y Hernández, F. (2018). El Aprendizaje Basado En Proyectos, una experiencia en las prácticas industriales del programa de Ingeniería Industrial de la UFPS. https://www.acofipapers.org/index.php/eiei/article/download/365/363/705

León, O., Martínez, L. y Santos, M. (2018). Análisis de la investigación sobre Aprendizaje basado en Proyectos en Educación Física. Revista Electrónica Interuniversitaria de $\begin{array}{llll}\text { Formación del 21(2), Profesorado, 27-42. } & \text { 21 }\end{array}$ http://dx.doi.org/10.6018/reifop.21.2.323241

López, N. (2017). Aprendizaje basado en proyectos (ABP) aplicado en Gestión Integral de Cuencas Hidrográficas. Caso de Estudio en Ingeniería Forestal. Bosques Latitud Cero, 7(2), 116-129. https://revistas.unl.edu.ec/index.php/bosques/article/view/325/298

Maldonado, M. (2008). Aprendizaje Basado en Proyectos Colaborativos. Una experiencia en $\begin{array}{llll}\text { educacion } \quad \text { superior. } & \text { Laurus, } & 14(28), & 158-180 .\end{array}$ https://www.redalyc.org/articulo.oa?id=76111716009

Manresa, A., Gil, D. y Berbegal, J. (2020). Adecuando el aprendizaje a los requerimientos de la empresa: una actividad de aprendizaje basado en proyectos. Revista d'Innovació Docent Universitària, (12), 54-61. http://dx.doi.org/10.1344/RIDU2020.12.6 
Martí, E., Gil, D., Vivet, M. y Julià, C. (2009). Aprendizaje Basado en Proyectos en la asignatura de Gráficos por Computador en Ingeniería Informática. Balance de cuatro años de experiencia, (XV), 8-10. http://jenui2009.fib.upc.edu

Martí, J., Heydrich, M., Rojas, M. y Hernández, A. (2010). Aprendizaje basado en proyectos: una experiencia de innovación docente. Revista Universidad EAFIT, 46(158), 11-21. https://publicaciones. eafit.edu.co/index.php/revista-universidadeafit/article/view/743

Martín, S. (2020). Aplicación de las Metodologías Ágiles al proceso de enseñanza aprendizaje universitario. Revista d'Innovació Docent Universitària, (12), 62-73. https://doi.org/10.1344/RIDU2020.12.7

Medina, J., Quintana, L. y García, A. (2017). Aprendizaje basado en problemas y proyectos para el fortalecimiento de capacidades y habilidades de los estudiantes de la UNAD: Caso de la fábrica de café y chocolate El Agrario, de San Vicente de Chucurí, Santander, Colombia. Revista Docencia Universitaria, 18(2), 69-79. https://revistas.uis.edu.co/index.php/revistadocencia/article/view/9036/9849

Medina-Nicolalde, M. y Tapia-Calvopiña, M. (2017). El Aprendizaje Basado en Proyectos una oportunidad para trabajar interdisciplinariamente. OLIMPIA, Revista de la Facultad de Cultura Física de la Universidad de Granma. 14(46). https://dialnet.unirioja.es/descarga/articulo/6220162.pdf

MEN. (s.f). Fortalecimiento a la educación media. https://www.mineducacion.gov.co/1759/w3-article-374737.html?_noredirect=1

Mora, W., Carranza, L., y Palíz, C. (2019). El aprendizaje basado en proyecto: Realidad y perspectivas. Revista Ciencia E Investigación, 4(4), 22-33. https://revistas.utb.edu.ec/index.php/sr/article/view/687

Mujica, A. (2012). Aprendizaje por proyectos: Una vía al fortalecimiento de los semilleros de investigación. Docencia Universitaria, (13), 201-216. https://revistas.uis.edu.co/index.php/revistadocencia/article/view/3268

Olarte, J. (2020). Homogeneizar la práctica de la modelación: un reto del sistema educativo colombiano. Revista Educación, 44(1). https://doi.org/10.15517/REVEDU.V44I1.36285

Parra, J., Amariles, M. y Castro, C. (2016). Aprendizaje basado en problemas en el camino a la innovación en ingeniería. Ingenierías USBMed, 7(2), 1-20. https://dialnet.unirioja.es/descarga/articulo/6007713.pdf 
Pressman, R. (1988). Ingeniería del software: Un enfoque práctico. McGraw-Hill.

Queiruga, M., Sáiz, M. y Montero, E. (2019). Problemas-Proyectos Adaptativos y Creativos en la enseñanza de las ciencias. Descripción de la metodología y apreciación de los estudiantes involucrados. Research in Education and Learning Innovation Archives, (23), 1-23. https://doi.org/10.7203/realia.23.15567

Rico, B., Garay, L. y Ruiz, E. (2018). Implementación del aprendizaje basado en proyectos como herramienta en asignaturas de ingeniería aplicada. RIDE Revista Iberoamericana Para La Investigación Y El Desarrollo Educativo, 9(17), 20 - 57. https://doi.org/10.23913/ride.v9i17.372

Rodríguez, E., Vargas, É. y Luna, J. (2010). Evaluación de la estrategia "aprendizaje basado en proyectos". Educación y Educadores, 13(1), 1-18. https://educacionyeducadores.unisabana.edu.co/index.php/eye/article/view/1618/2 128

Rodríguez, F., Kolmos, A., y Guerra, A. (2017). Aprendizaje basado en problemas en ingeniería: Teoría y práctica. https://www.acofipapers.org/index.php/eiei/article/download/365/363/705

Rodríguez-Sandoval, E. y Cortés-Rodriguez, M. (2010). Evaluación de la estrategia pedagógica "aprendizaje basado en proyectos": percepción de los estudiantes. Revista da Avaliação da Educação Superior, 15(1), 143-158. https://doi.org/10.1590/S1414-40772010000100008

Romero, A., Forero, A. y Rodríguez, A. (2018). Análisis comparación del aprendizaje basado en proyectos de forma tradicional y con mediación de las TIC. Revista Espacios, 39(52), 28. http://www.revistaespacios.com/a18v39n52/a18v39n52p28.pdf

Sánchez, L., Ferrero, R., Conde, M. y Alfonso, J. (2016). Desarrollo de competencias emprendedoras mediante iniciativas de aprendizaje basado en proyectos. Education in the Knowledge Society (EKS), 17(4), 15-28. https://doi.org/10.14201/eks20161741528

Sánchez, P. y Blanco, C. (2012). Implantación de una metodología de aprendizaje basada en proyectos para una asignatura de Ingeniería del Software. https://core.ac.uk/download/pdf/41791858.pdf

Santos, M. (2015) Corazones, no solo cabezas en la universidad. Los sentimientos de los estudiantes ante la evaluación. Revista de Docencia Universitaria, 13(2), 125-142. https://doi.org/10.4995/redu.2015.5441 
Toledo, P. y Sánchez, J. (2018). Aprendizaje Basado en Proyectos: Una Experiencia Universitaria. Revista Profesorado, 22(2). https://doi.org/10.30827/profesorado.v22i2.7733

UCEVA. (2013). Proyecto Educativo del Programa de Ingeniería de Sistemas de la UCEVA. https://www.uceva.edu.co/wpcontent/uploads/2020/09/PEP_Proyecto_educativo_programa_de_ingenieria_de_sis temas-1.pdf

Unipamplona. (2018). Proyecto educativo del Programa de ingeniería de sistemas. Facultad de ingenierías y arquitectura, Universidad de Pamplona. http://www.unipamplona.edu.co/unipamplona/portalIG/home_77/recursos/docume ntos/12122018/2018pepingsistemas.pdf

Valbuena, S., Muñiz, L. y Berrío, J. (2020). El rol del docente en la argumentación matemática de estudiantes para la resolución de problemas. Revista Espacios, 41(9), 1-25. https://www.revistaespacios.com/a20v41n09/20410915.html

Vélez, J. y Benjumea, P. (2017). Estrategia de Innovación en Educación en Ingeniería. https://tyche.medellin.unal.edu.co/descargas/InnovacionenEducacionenIngenieria \%20FM.pdf

Villalobos-Abarca, et al. (2018). Aprendizaje Basado en Proyectos Reales Aplicado a la Formación del Ingeniero de Software. Formación universitaria, 11(3), 97-112. https://dx.doi.org/10.4067/S0718-50062018000300097

Villavicencio, E. (2017). El tamaño muestral para la tesis ¿Cuántas personas debo encuestar? Revista Oactiva Uc-Cuenca, 2(1), 59-62. https://doi.org/10.31984/oactiva.v2i1.175 


\section{Anexo I \\ Encuesta para la evaluación del ABPrj aplicado como herramienta en la asignatura de IPOO - Universidad del Valle sedes Buga y Tuluá}

Califique de 1 a 5, donde 1 es la nota mínima y 5 la máxima, cada uno de los siguientes interrogantes.

\begin{tabular}{|c|c|c|}
\hline \multicolumn{3}{|c|}{ Presentación del proyecto } \\
\hline \# & Pregunta & Calificación \\
\hline 1 & El objetivo del proyecto está claramente definido & \\
\hline 2 & $\begin{array}{l}\text { El objetivo del proyecto está relacionado con el contenido curricular del programa } \\
\text { académico }\end{array}$ & \\
\hline 3 & $\begin{array}{l}\text { El objetivo del proyecto me permitió aplicar conceptos de otras asignaturas o áreas } \\
\text { del conocimiento para su solución }\end{array}$ & \\
\hline 4 & En el enunciado del proyecto se describe cual debe ser el producto final & \\
\hline \multicolumn{3}{|c|}{ Desarrollo de competencias específicas } \\
\hline 5 & $\begin{array}{l}\text { Para el desarrollo del proyecto aplique los conceptos adquiridos en la asignatura de } \\
\text { IPOO }\end{array}$ & \\
\hline 6 & El proyecto me permitió relacionar los conceptos teóricos y llevarlos a la practica & \\
\hline 7 & $\begin{array}{l}\text { El desarrollo del proyecto aumento el interés por la asignatura de IPOO y la } \\
\text { programación de computadoras }\end{array}$ & \\
\hline 8 & El desarrollo del proyecto aportó a mi formación profesional & \\
\hline \multicolumn{3}{|c|}{$\begin{array}{c}\text { Desarrollo de competencias generales: } \\
\text { a. Instrumentales }\end{array}$} \\
\hline 9 & $\begin{array}{l}\text { El desarrollo del proyecto permitió adquirir habilidades para la organización y } \\
\text { planificación del tiempo }\end{array}$ & \\
\hline 10 & $\begin{array}{l}\text { El desarrollo del proyecto permitió adquirir habilidades para la resolución de } \\
\text { problemas }\end{array}$ & \\
\hline 11 & El desarrollo del proyecto permitió adquirir habilidades para la toma de decisiones & \\
\hline 12 & $\begin{array}{l}\text { Para el desarrollo del proyecto aplique los conceptos adquiridos e investigados en } \\
\text { otras asignaturas del currículo y áreas del conocimiento }\end{array}$ & \\
\hline \multicolumn{3}{|c|}{ b. Sistémicas } \\
\hline 13 & El desarrollo del proyecto incentivo mi creatividad & \\
\hline 14 & $\begin{array}{l}\text { El enfoque del proyecto me permitió establecer tareas por objetivos para su } \\
\text { desarrollo }\end{array}$ & \\
\hline 15 & $\begin{array}{l}\text { El enfoque del proyecto me permitió establecer un plan de actividades para su } \\
\text { desarrollo }\end{array}$ & \\
\hline 16 & $\begin{array}{l}\text { El desarrollo del proyecto incentivo la investigación de temas relacionados con los } \\
\text { requisitos del proyecto }\end{array}$ & \\
\hline 17 & $\begin{array}{l}\text { El enfoque del proyecto me permitió delegar tareas a mis compañeros de equipo de } \\
\text { trabajo }\end{array}$ & \\
\hline \multicolumn{3}{|c|}{ c. Interpersonales } \\
\hline 18 & La metodología del proyecto incentivo mi automotivación & \\
\hline 19 & El desarrollo del proyecto incentivo las relaciones y la comunicación interpersonal & \\
\hline 20 & El desarrollo del proyecto incentivo el trabajo en equipo & \\
\hline 21 & $\begin{array}{l}\text { La metodología del proyecto me permitió adquirir habilidades para la resolución de } \\
\text { conflictos y negociación ante problemas en el equipo de trabajo }\end{array}$ & \\
\hline 22 & $\begin{array}{l}\text { El desarrollo del proyecto me permitió aplicar características de liderazgo ante los } \\
\text { integrantes del equipo de trabajo }\end{array}$ & \\
\hline
\end{tabular}

